\title{
Performance Evaluation and QoS Analysis of BCDCP and CBCB Routing Protocols in Wireless Sensor Networks
}

\author{
Sangeeta Vhatkar \\ Assistant Professor, TCET \\ Ph.D. Scholar \\ P.I.E.T, Nagpur
}

\author{
Srishti Mehta \\ M.E I.T \\ Thakur College of Engineering \\ and Technology \\ University of Mumbai
}

\author{
Mohammad Atique \\ 3rd Associate Professor \\ P.G Department of Computer \\ Science, S.G.B.A.U \\ Amravati
}

\begin{abstract}
A wireless sensor network (WSN) consists of low power multifunctioning sensor nodes which operate in an unattended environment with limited computational and sensing capabilities. Once deployed, the small sensor nodes are usually inaccessible to the user, and thus replacement of the energy source is not feasible. Therefore, energy efficiency is a key design issue that needs to be enhanced in order to improve the life span of the network. The sensor nodes communicate with each other via various Routing Protocols. Base-Station Controlled Dynamic Clustering Protocol (BCDCP) is a hierarchical routing protocol that distributes the energy dissipation evenly among all sensor nodes to improve network lifetime and average energy savings. To overcome the drawbacks of the BCDCP protocol, in this research a new enhanced and improved BCDCP protocol called the mobileBCDCP (M-BCDCP) protocol has been proposed. The simulation result compares the M-BCDCP protocol with the improvised $\mathrm{CBCB}$ protocol and shows that M-BCDCP protocol has enhanced performance than $\mathrm{CBCB}$ protocol in terms of number of packets delivery, energy consumption, delay, network lifetime and throughput.
\end{abstract}

\section{General Terms}

Routing protocols, QoS, wireless sensor network, hierarchical routing

\section{Keywords}

WSN, hierarchical routing protocols, M-BCDCP, QoS, $\mathrm{CBCB}$, energy consumption, delay, packet delivery ratio, throughput, network lifetime

\section{INTRODUCTION}

Thousands of low-power sensor nodes are deployed in an unattended environment which makes up a wireless sensor network (WSN). These sensor nodes have limited sensing and computational capabilities and once deployed, they usually become inaccessible to the user. Thus, replacement of energy is not feasible. Recent developments in WSN have made the sensor nodes small in size and low in cost. Hence, energy efficiency is a key design issue that needs to be enhanced in order to improve the life span of the network. Several network layer protocols have been proposed to improve the effective lifetime of a network with a limited energy supply. Most of the attention, however, has been given to the routing protocols since they might differ depending on the application and network architecture [[1], [2]].

Sensor nodes can sense, process, transmit, mobilize and receive power using optical components. These nodes coordinate among themselves to generate finest information about the physical environment. The decision of every node is established on its mission, the information it currently has, knowledge of its computing, communication and energy resources. These sensor nodes have capability to transmit the sensed information among each other or to the base station (BS) [3]. Every node has limited amount of energy, communication capacity and calculating power. Hence optimizing the correct communication path, losing minimum number of packets while transmitting the data, having minimum delay in transmission, consuming least energy and providing higher end-to-end performance are the main issues while choosing the routing protocol. Some applications of sensor nodes are in the field of military and security, environmental monitoring, automobile industries, patient health monitoring, constructions, etc [[4], [5]].

Hierarchical Routing protocols or cluster based routing protocols divides the nodes into clusters. Hierarchical-based routing is prominent for achieving empirically good and promising results in terms of network lifetime and energy savings. This is attributed to the fact that, as means of reducing data traffic and consequently minimizing the amount of energy dissipated, hierarchical based routing protocols perform local data processing and aggregation as early as possible. Each cluster randomly selects a node that acts as a cluster head $(\mathrm{CH})$. Different protocols under hierarchical routing mainly fluctuate from the process of selection of $\mathrm{CH}$ and behavior of every node [6].

Low Energy Adaptive Clustering Hierarchy (LEACH) and Power Efficient Gathering in Sensor Information Systems (PEGASIS) are two typical hierarchical protocols. LEACH randomizes the rotation of local $\mathrm{CH}$ to evenly distribute the energy load among the sensors in the network. It localizes the coordination to enable the scalability and strength for the dynamic networks. It performs data fusion while routing so that amount of information transmitted to BS is reduced and bandwidth is saved [7]. PEGASIS is a near optimal chain based routing protocol where each node only communicates with the nearest node and takes turns while transmitting the data to the BS, hence saving the amount of energy consumed in each round [[8], [9]].

As an extension of LEACH and PEGASIS, a centralized cluster-based routing protocol, called Base-Station Controlled Dynamic Clustering Protocol (BCDCP) is a wireless sensor routing protocol with the base station being an essential component with complex computational abilities, thus making the sensor nodes very simple and cost effective. In BCDCP, energy intensive computation decisions are taken by the BS, 
which is assumed to be non-energy constrained and to be fully aware of the location of all sensor nodes in the network. BCDCP builds uniformly distributed clusters; shielded from getting confined to a particular region in the network. It performs balanced cluster formation by balancing the size of the clusters. BCDCP provides drastic improvements over LEACH, LEACH-C and PEGASIS. It avoids direct transmission from each $\mathrm{CH}$ to the distant BS which yields substantial reduction in energy consumption. Also clusters are no longer isolated from each other, and so the death of one $\mathrm{CH}$ would have a profound effect on partitioning the network and causing other $\mathrm{CHs}$, along with all the nodes in their clusters, to be virtually dead [[10], [11], [12]].

Though BCDCP protocol has its advantages over LEACH and PEGASIS protocol, it still had certain deficiencies. There are several protocols that are developed on the basis of BCDCP such as DMSTRP [13], ADCP [14], CBCB [15], ICRP [16], SLDHP [17], DEEHRP [18] et.al. Each protocol proposes and takes into consideration unique factors.

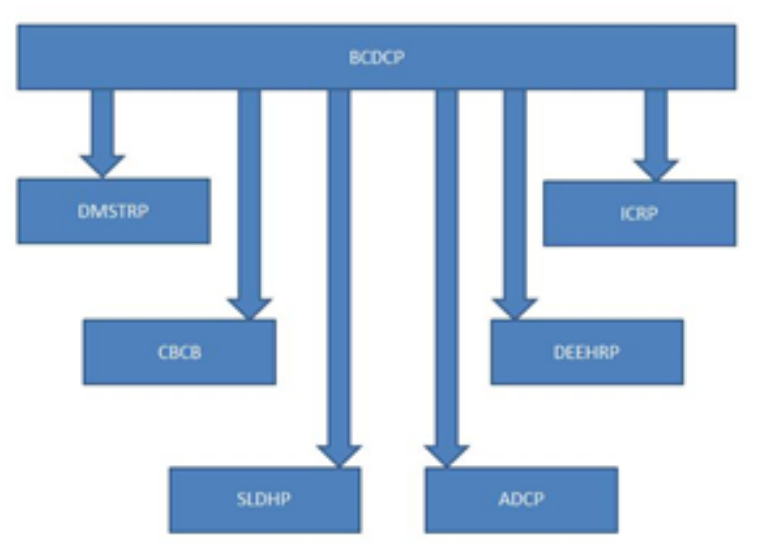

Fig 1: Various BCDCP based protocols

The proposed M-BCDCP protocol uses the concept of mobile nodes. It enables the nodes to reach the BS directly which was previously the drawback of BCDCP protocol. The M-BCDCP protocol forms regions to divide the nodes and then does cluster formation within the regions which reduces the complexity of data transfer. Also improved results for CBCB protocol have been seen in [19]. In this paper, comparison of these two improved protocols is done in terms of the various Quality of Service (QoS) parameters like delay, throughput, packet delivery ratio, network lifetime and energy consumption. Simulation results show that M-BCDCP outperforms $\mathrm{CBCB}$ in all parameters. The rest of the paper is organized as follows: Section 2 describes M-BCDCP and CBCB protocols. Section 3 explains the methodology of MBCDCP protocol. Network model and steps of execution for the M-BCDCP protocol are mentioned in Section 4. Finally Section 5 shows the simulation of M-BCDCP and $\mathrm{CBCB}$ protocol. Section 6 concludes the proposed work and offers future directions.

\section{DESCRIPTION OF BCDCP AND CBCB PROTOCOL \\ 2.1 BCDCP}

$\mathrm{BCDCP}$ is a centralized cluster-based routing protocol. In BCDCP, energy intensive computation decisions are held by the BS, which is assumed to be non - energy constrained. The $\mathrm{BS}$ is also assumed to be fully aware of the location of all sensor nodes in the network. BCDCP builds uniformly distributed clusters; shielded from getting confined to a particular region in the network. It performs balanced cluster formation by balancing the size of the clusters [11].

During data-communication phase, each $\mathrm{CH}$ receives signals from the non-cluster-head members, and aggregates them before engaging in a multi-hop $\mathrm{CH}$-to- $\mathrm{CH}$ routing path, constructed by applying minimum spanning tree (MST) algorithm. The minimum spanning tree technique applied in BCDCP connects all $\mathrm{CHs}$ based on their spatial separation, so as to minimize the amount of energy dissipated for each $\mathrm{CH}$. Upon constructing the minimum spanning tree, BCDCP randomly selects one of the $\mathrm{CHs}$ to be the one that forwards the data to the distant BS. This random choice is motivated by the desire to resolve the hot spot problem. BCDCP avoids direct transmission between each $\mathrm{CH}$ to the distant $\mathrm{BS}$ and therefore yields substantial reduction in energy consumption.

It is well known and understood that the most important part in sensor networks is the life span of the nodes. Each node in a sensor network would become useless after wasting its energy completely since its power is totally dependent on the embedded battery which is unlikely to be recharged due to the remoteness of the area. BCDCP is an energy efficient routing protocol that is used to extend the life span of the nodes in the networks. In sensor networks, once a node starts to die then the whole network is considered to be dead since the first node would trigger all the others nodes to die shortly after. The objective of $\mathrm{BCDCP}$ protocol is to make the network energy-efficient and fault tolerant. BCDCP is a centralized routing protocol where $\mathrm{BS}$ has the maximum energy and computing power and knows all node locations and therefore does the balanced cluster formation. BS also creates the TDMA schedule and forms the $\mathrm{CH}$ spanning tree. Only one $\mathrm{CH}$ forwards to the BS [[10], [11]].

\subsection{CBCB}

A Centralized Border Node based Cluster Balancing (CBCB) protocol evenly distributes the load equally among the clusters to improve network lifetime. The main idea in CBCB is to maintain balanced number of sensor nodes among each cluster to prevent the overloading of $\mathrm{CH}$, selection of $\mathrm{CHs}$ to cover the entire sensing area and use of multi-hop for head-to-head routing for forwarding the sensed information to remote BS. This protocol makes use of a BS with high energy in order to form clusters and discover the paths in routing, the $\mathrm{CH}$ rotation and to perform the different jobs which need intense energy [15].

\section{MEHTODOLOGY FOR M-BCDCP PROTOCOL}

M-BCDCP protocol works in two stages: 1) Setup Phase 2) Data Communication Phase.

\subsection{Setup Phase}

- A $1350 \times 1100 \mathrm{~m}$ network area in which 100 nodes are densely deployed has been considered.

- Initialize all the network parameters.

- Determine the number of nodes, initial energy and BS location.

- Form the three regions in the network area.

- Set the nodes at various specified locations - cluster formation in these three regions. 
a) Choose the two most separated nodes as the $\mathrm{CHs}$.

b) Then based on the proximity to these selected $\mathrm{CHs}$, divide the nodes.

c) Balance the clusters i.e. make equal nodes on each side.

d) Iterate this process to divide the remaining nodes and form clusters accordingly.

- There are two types of nodes - mobile and static.

- Accordingly the user can select the source and destination from these two types of nodes.

- Each node calculates a known distance from the origin as well as other neighboring nodes - neighbor calculation.

- Give the color, label and shape to all the nodes.

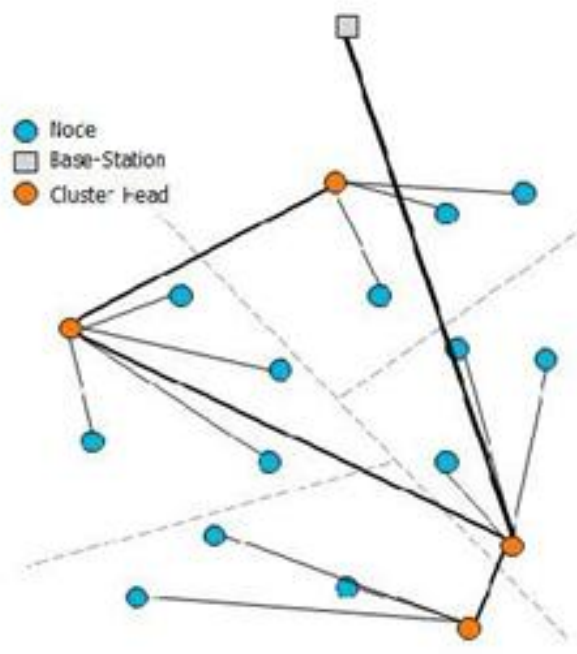

Fig 2: M-BCDCP protocol after Setup and Data Communication Phase

\subsection{Data Communication Phase}

- In this phase, actual data is transmitted from source to destination.

- Using the TDMA schedule, nodes send data to the cluster head.

- Once it finds the result from the node selected, it takes the minimum distance result from the neighbor node. (MST used)

- Then it starts sending data packet to the nearest neighbor node (from the neighbor calculation table).

- $\mathrm{CH}$ performs data fusion.

- Compressed data is then routed to the base-station.

- Then this data is forwarded to the chosen destination.

- The source and destination can be in same or different regions according to user's chosen node.

- In the data transmission if any case data replication occurred then the same amount of data should be transmit more than one time. By this, the system power, efficiency and other factors may be affected.

- To resolve this problem, we initialize the collision bit rate (CBR) traffic creation.

- Here only one intermediate node among the participated node is considered into the transmission and give the same amount of data.

- By this it will send this data to the destination again. Therefore there is no chance of replication or redundancy.

Figure 3 shows the flowchart for working of the M-BCDCP protocol.

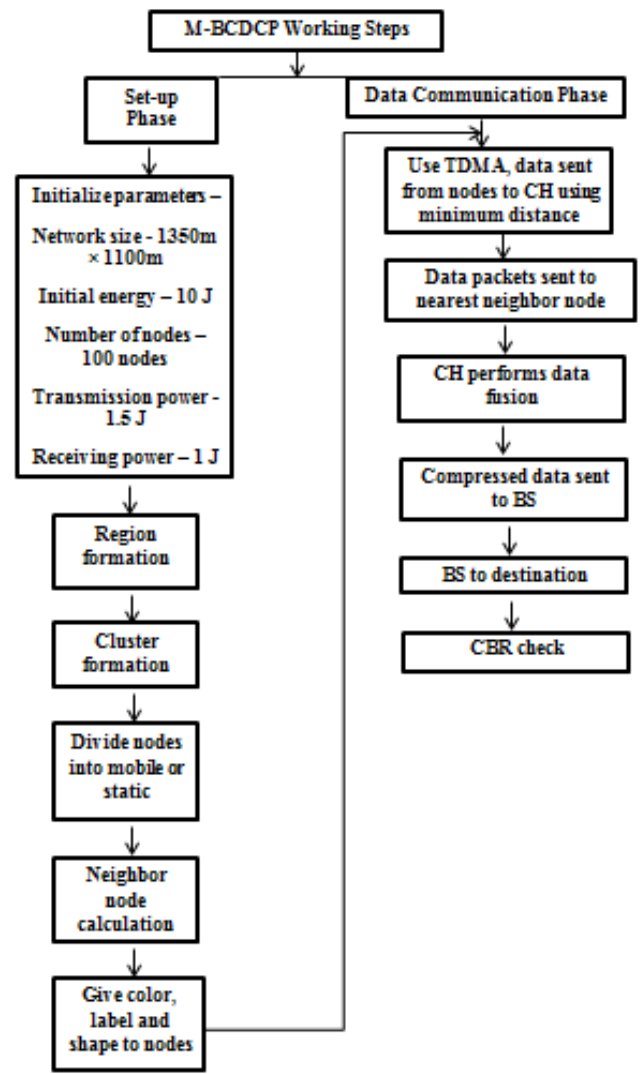

Fig 3: Flowchart for working of M-BCDCP protocol

\section{NETWORK MODEL AND STEPS OF ENHANCED BCDCP PROTOCOL}

\subsection{Network Parameters}

In this paper a simple WSN model is assumed with following properties:

- $\quad \mathrm{N}$ number of sensor nodes are randomly distributed in a field where $\mathrm{N}=100$

- Sensor nodes are both mobile and static.

- Sensor nodes are standardized in terms of initial energy, receiving power, transmitting power and sensing power.

- BS is located in between the field and does not have any energy constraints. 
Table 1 shows the values assigned to different network parameters.

Table 1. Network Parameter Values

\begin{tabular}{|c|c|}
\hline Parameters & Values \\
\hline Network Size & $1350 \times 1100$ meter $^{2}$ \\
\hline Number of Nodes & 100 nodes \\
\hline Initial Energy of Nodes & $10 \mathrm{j}$ \\
\hline Transmission Power & $1.5 \mathrm{j}$ \\
\hline Receiving Power & $1 \mathrm{j}$ \\
\hline Idle Power & $1.2 \mathrm{j}$ \\
\hline Sleep Power & $0.000015 \mathrm{j}$ \\
\hline
\end{tabular}

\section{SIMULATION OF BCDCP AND \\ CBCB PROTOCOL - \\ PERFORMANCE EVALUATION}

This paper uses NS - 2.35 simulator to evaluate the performance of the M-BCDCP protocol by comparing it with $\mathrm{CBCB}$ protocol. Performance is measured on various QoS parameters such as Packet Drop Ratio, Delay, Throughput, Network Lifetime and Energy Consumption. Throughout the simulations, we consider 100 nodes network configuration where each node is assigned an initial energy of $10 \mathrm{~J}$. The network size used is $1350 \times 1000 \mathrm{~m}^{2}$. Based on the parameters mentioned in Table 1, simulate the end-to-end performance of the $\mathrm{M}-\mathrm{BCDCP}$ protocol and compare it with $\mathrm{CBCB}$ protocol.

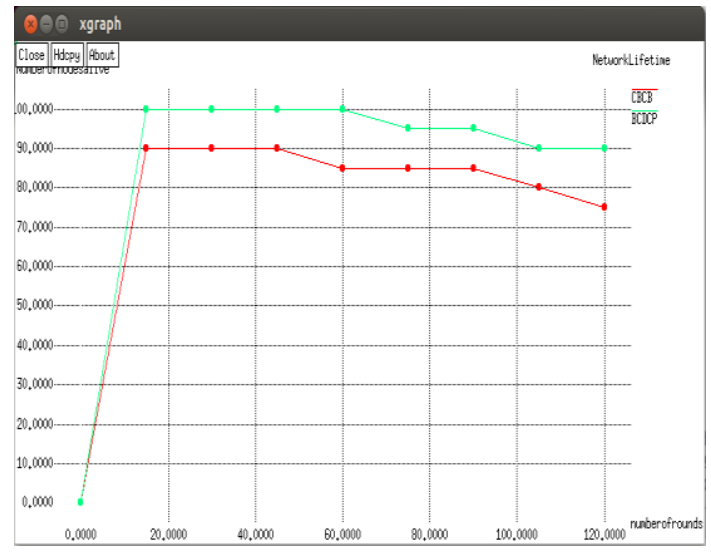

Fig 4: a) Network Lifetime

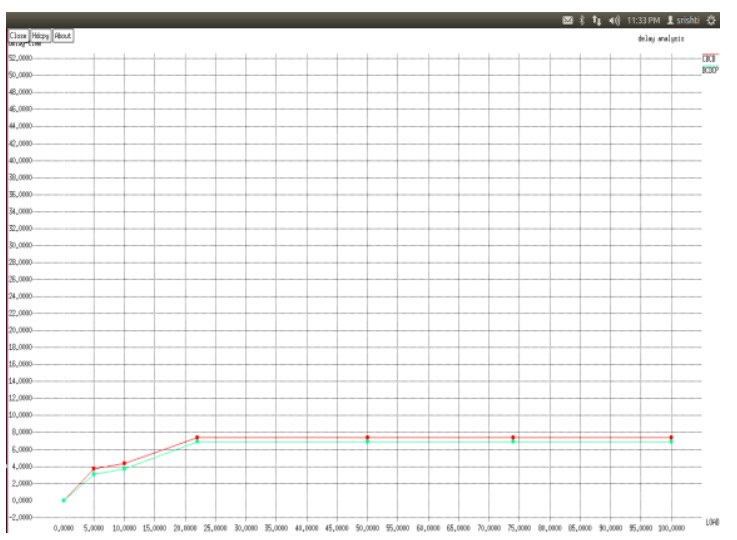

Fig 4: b) Delay

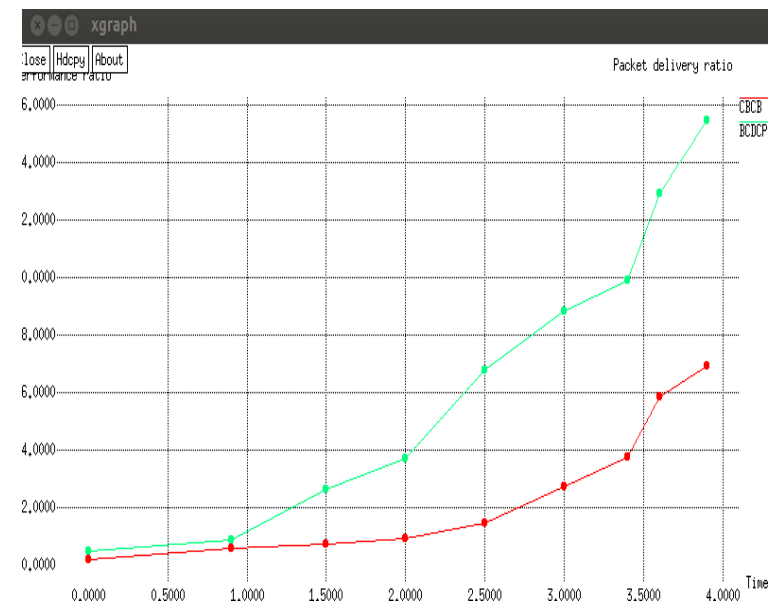

Fig 4: c) Packet Delivery Ratio

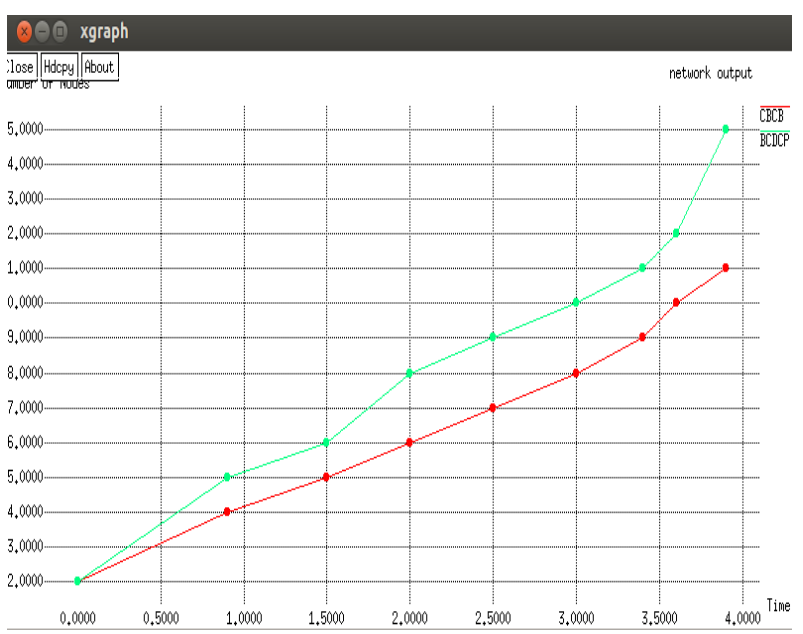

Fig 4: d) Network Output

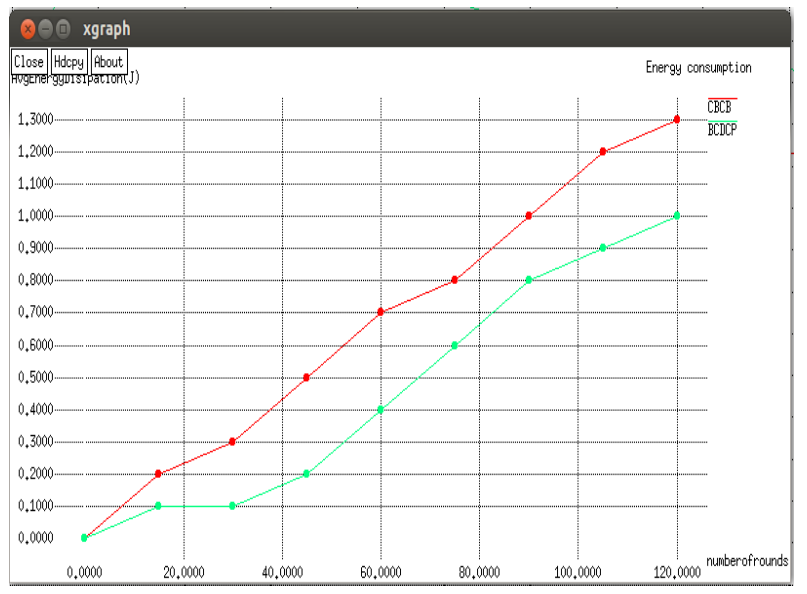

Fig 4: e) Energy Consumption

The Fig 4: a) shows the network lifetime graph for M-BCDCP and $\mathrm{CBCB}$ protocol. It shows how $\mathrm{M}-\mathrm{BCDCP}$ is increasing the overall network life when compared with the $\mathrm{CBCB}$ protocol. With M-BCDCP, 90 nodes remain alive out of 100 for 120 rounds whereas in $\mathrm{CBCB}$, only 75 nodes remain alive for the same. This shows that M-BCDCP has $16.7 \%$ more network life than $\mathrm{CBCB}$ routing protocol.

The Fig 4: b) graphs the delay in terms of load (number of packets) with respect to time. For the maximum number of 
packets transferred, the M-BCDCP protocol has $6.76 \%$ less delay than CBCB protocol.

The Fig 4: c) shows the packet delivery ratio of both the protocols. It is clearly seen that $\mathrm{CBCB}$ drops more packets than the M-BCDCP protocol. For maximum number of packets transferred the M-BCDCP has $55.23 \%$ more packet delivery ratio than $\mathrm{CBCB}$. Thus it is concluded that the MBCDCP out performs CBCB in terms of packet delivery.

As shown in the Fig 4: d) network output graph will calculate the throughput of the network. M-BCDCP protocol outdoes the $\mathrm{CBCB}$ protocol in it as well. Throughput is measured in terms of number of packets delivered with respect to time. For maximum number of packets transferred, M-BCDCP protocol has $26.67 \%$ more throughput than CBCB. Thus the M$\mathrm{BCDCP}$ has better throughput than CBCB.

The Fig 4: e) indicates the energy consumption of both the protocols under study over number of rounds of operation. Due to the region formation, only the cluster heads of regions communicate and forward the data to the base station as well as destination node. This in turn reduces the energy consumed for $\mathrm{M}-\mathrm{BCDCP}$ over $\mathrm{CBCB}$ protocol. For the maximum number of packets transferred, M-BCDCP consumes $23.07 \%$ less energy than $\mathrm{CBCB}$. This determines that the M-BCDCP surpasses CBCB in terms of saving energy as well.

Fig 5 shows the overall comparisons of M-BCDCP and CBCB protocol. M-BCDCP clearly shows improvement in all the QoS parameters reviewed over the $\mathrm{CBCB}$ routing protocol.

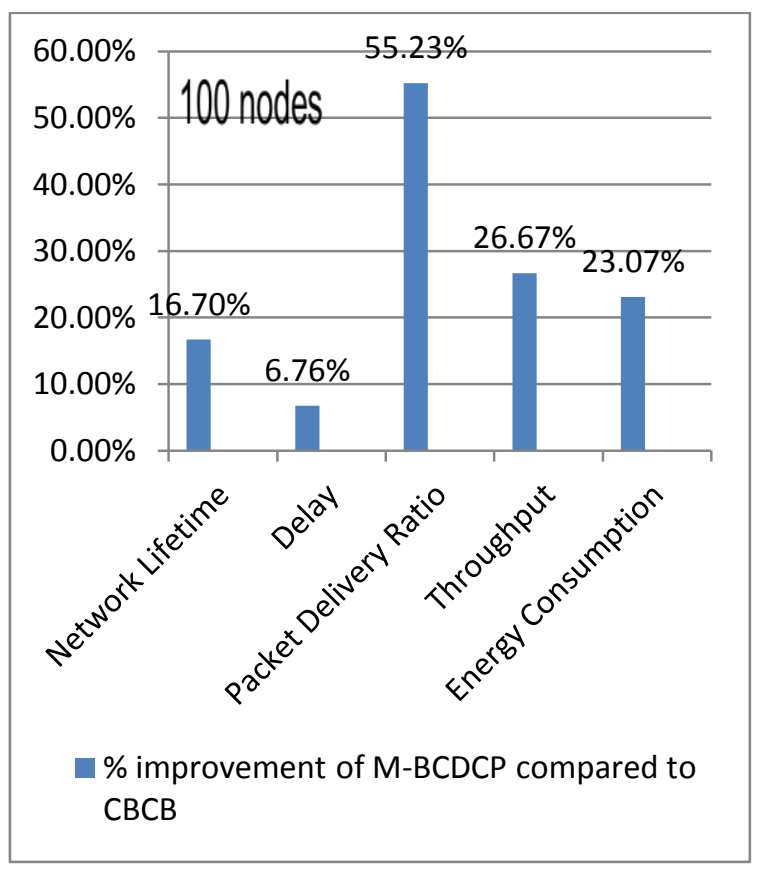

Fig 5: Overall Comparison of M-BCDCP to CBCB protocol

\section{CONCLUSION AND FUTURE WORK}

This paper describes the methodology of the new improved BCDCP protocol i.e. the M-BCDCP in details. Unlike CBCB, M-BCDCP works better in all aspects due to the concept of building regions and then transmitting the data from the source to the destination using mobile nodes. Comparison between $\mathrm{M}-\mathrm{BCDCP}$ and $\mathrm{CBCB}$ is performed and QoS is improvised. On the basis of derived results, it is depicted that enhanced M-BCDCP outperforms CBCB by $55.23 \%, 23.07$ $\%, 6.76 \%, 16.7 \%$ and $26.67 \%$ in terms of packet delivery ratio, energy consumption, delay, network lifetime and throughput respectively. Thus it is concluded that M-BCDCP has better end-to-end performance than $\mathrm{CBCB}$ protocol. In future, the M-BCDCP protocol can be compared with other BCDCP based protocols and QoS can be analyzed.

\section{REFERENCES}

[1] F. Akyildiz et al., "Wireless Sensor Networks: A Survey," Elsevier Sci. B.V. Comp. Networks, vol. 38, no. 4, Mar. 2002, pp. 393-422.

[2] W.Heinzelman, "Application Specific Protocol Architecture for Wireless Sensor Network", PhD Thesis, Massachusetts institute of technology, June 2000

[3] J. Al-Karaki, and A. Kamal, "Routing Techniques in Wireless Sensor Networks: A Survey", IEEE Communications Magazine, vol. 11, no. 6, pp. 6-28, Dec. 2004

[4] I.F. Akyildiz, W. Su, Y. Sankarasubramaniam, and E. Cayirci, "Wireless Sensor Network: A Survey" Computer Networks, pp. 393-22, March 2002.

[5] F. Akyildiz, W. Su, Y. Sankarasubramaniam, and E. Cayirci, "A Survey on Sensor Networks," IEEE Communications Magazine, Aug. 2002

[6] S. Singh, M. Singh, D. Singh, "A Survey of EnergyEfficient Hierarchical Cluster-Based Routing in Wireless Sensor Networks", International Journal of Advanced Networking and Applications Volume: 02, Pages: 570580,2010

[7] W. R. Heinzelman, A. Chandrakasan, and H. Balakrishnan, "Energy Efficient Communication Protocol for Wireless Microsensor Networks", in IEEE Computer Society Proceedings of the Thirty Third Hawaii International Conference on System Sciences (HICSS '00), Washington DC, USA, vol. 8, pp. 8020, Jan. 2000

[8] S. Lindsey and C. S. Raghavendra, "PEGASIS: Power Efficient Gathering in Sensor Information Systems," in IEEE Aerospace Conference, March 2002.

[9] Vhatkar S. and Atique M., "Design Issues and Challenges in Hierarchical Routing Protocols for Wireless Sensor Networks," Computational Science and Computational Intelligence (CSCI), 2014 International Conference on 10-13 March 2014, Las Vegas, NV, IEEE, March 2014, vol. 1, pp. 90-95

[10] Siva D. Muruganathan, Daniel C. F. MA, Rolly I. Bhasinand Abraham O. Fapojuwo, "A Centralized Energy-Efficient Routing Protocol for Wireless Sensor Networks", Communications Magazine, IEEE, vol.43,no. 3, pp. 8-13, March 2005.

[11] S. Lindsey, C. Raghavendra, and K. M. Sivalingam, "Data Gathering Algorithms in Sensor Networks using Energy Metrics," IEEE Trans. Parallel and Distrib. Sys., vol. 13, no. 9, Sept. 2002, pp. 924-35.

[12] Sara Abd EL Aziz, Haitham S. Hamza, Imane A. Saroit, "An Energy-Efficient Hierarchical Routing Protocol for Wireless Sensor Networks", Informatics and Systems 
(INFOS), 2010 The $7^{\text {th }}$ International Conference on 28 30 March 2010, Cairo, IEEE, pp. 1 - 7.

[13] Guangyan Huang, Xiaowei Li and Jing He, "Dynamic Minimal Spanning Tree Routing Protocol for Large Wireless Sensor Networks," Industrial Electronics and Applications, 2006 1ST IEEE Conference on 24-26 May 2006, Singapore, IEEE Conference Publications, May 2006, pp. 1-5

[14] Bansal, T., Ghanshani, P., Joshi, R.C., “An Application Dependent Communication Protocol for Wireless Sensor Networks," Networking, International Conference on Systems and International Conference on Mobile Communications and Learning Technologies, 2006. ICN/ICONS/MCL 2006. International Conference on 2329 April 2006, IEEE Conference Publications, April 2006, pp. 120

[15]Krishna B B, A.S Raghuvanshi, "Centralized Border Node based Cluster Balancing Protocol for Wireless Sensor Networks ," Contemporary Computing (IC3), 2013 Sixth International Conference on 8-10 Aug. 2013, Noida, IEEE Conference Publications, pp. 35-40, Aug. 2013

[16]Nurhayati,"Inner Cluster Routing Protocol Wireless Sensor Network," Computer and Communication
Engineering (ICCCE), 2012 International Conference on 3-5 July 2012, Kuala Lampur, IEEE, July 2012, pp. 894898

[17] Tarannum, S Srividya, Asha D.S. , Padmini R., Nalini L., Venugopal K.R. and Patnaik L.M. "Dynamic Hierarchical Communication Paradigm for Wireless Sensor Networks: A Centralized, Energy Efficient Approach," Communication Systems, 2008. ICCS 2008. 11th IEEE Singapore International Conference on 19-21 Nov. 2008, Guangzhou, IEEE Conference Publications, Nov. 2008, pp. 959-963

[18] Abd El Hamid S.A. ,Hamza H.S., Saroit I.A. “Alleviating the Domino Effect in Wireless Sensor Networks," Local Computer Networks (LCN), 2013 IEEE 38th Conference on 21-24 Oct. 2013, Sydney, NSW, IEEE, Oct. 2013, pp. 256-259

[19] Srishti Mehta, Sangeeta Vhatkar and Mohommad Atique, "Comparative Study of BCDCP Protocols in Wireless Sensor Network," in International Journal of Computer Applications (IJCA) Proceeding on International Conference and Workshop on Emerging Trends in Technology (ICWET 2015), pp. 7-12, May 2015. 